\title{
Recurrent congenital diaphragmatic hernia: A single center experience
}

\author{
Żaneta Słowik-Moczydłowska, Andrzej Kamiński \\ Department of Paediatric Surgery, Medical University of Warsaw, Poland
}

\begin{abstract}
Congenital Diaphragmatic Hernia (CDH) occurs in 1:4000 live births. It's morbidity and mortality rates are significant. Recurrence is one of the recognized complications occurring after a successful initial repair. Poorly known are the rate of recurrence and factors that may influence the re-herniation. Poorly described are its morphology and treatment. Medical records of 95 neonates with $\mathrm{CDH}$ who had undergone repair at tertiary pediatric teaching hospital between 2007-2018 were retrospectively reviewed. Prior to surgery, peri-operative and recurrence data were collected. The follow-up was 1 to 12 years after the initial repair. Recurrence occurred in $22 / 84(26 \%)$ patients. In 5 patients we observed more than one recurrence, total number of 27 (32\%) re-herniations; $16 / 22(72 \%)$ recurrences occurred within first 12 months; 7/27 (26\%) recurrences were symptomatic which gives the symptomatic recurrence rate $8.3 \% ; 20 / 27(74 \%)$ were asymptomatic. There were no significant differences between groups considering LHR, gestational age, birth weight, severity of pre-surgery and post-surgery stabilization period. Significantly higher Apgar
\end{abstract}

Correspondence: : Żaneta Słowik-Moczydłowska; Department of Paediatric Surgery, Medical University of Warsaw, ul. Żwirki i Wigury 63A, 02-091 Warszawa, Poland.

Tel.: +48 223179736 .

E-mail: zaneta.moczydlowska@uckwum.pl

Key words: Congenital diaphragmatic hernia; $\mathrm{CDH}$ recurrence; $\mathrm{CDH}$ re-herniation.

Conflict of interest: The authors declare no conflict of interest.

Availability of data and materials: All data underlying the findings are fully available.

Ethics approval and consent to participate: All procedures performed in studies involving human participants were in accordance with the ethical standards of the Institutional Research Committee and with the 1964 Helsinki declaration and its latest amendment.

Consent for publication: Not applicable.

Received for publication: 4 March 2020.

Accepted for publication: 11 February 2021.

This work is licensed under a Creative Commons Attribution

NonCommercial 4.0 License (CC BY-NC 4.0).

CCopyright: the Author(s), 2021

Licensee PAGEPress, Italy

La Pediatria Medica e Chirurgica 2021; 43:228

doi:10.4081/pmc.2021.228 scores were in the group without recurrence. Among subjects with recurrent $\mathrm{CDH}$ majority had a left-sided defect, no hernia sac, no liver herniation, diaphragm was primarily sutured, abdominal wall primarily closed. Even though none of those factors was significantly related with the recurrence. Recurrence in $\mathrm{CDH}$ remains a significant issue for long-term surgical morbidity, especially more apparent in the first year of life. As long as the risk factors of reherniation remains unclear, the most important seems to be routine follow-up protocol allowing for $\mathrm{CDH}$ recurrence detection.

\section{Introduction}

Congenital Diaphragmatic Hernia $(\mathrm{CDH})$ occurs in approximately 1:4000 live births. It's morbidity and mortality rates are significant. ${ }^{1}$ Improvement in prenatal assessment, perinatal management and postnatal treatment have increased the survival rate and made long term fallow-up more apparent. ${ }^{2}$

Recurrence is one of the recognized but yet unpredictable complication occurring after a successful initial repair of $\mathrm{CDH}$. Still poorly known are the rate of recurrence, the exact factors that may influence the incidents of recurrence or that may predispose to re-herniation. Poorly described are even morphology and treatment of recurrent $\mathrm{CDH}$.

Multiple studies point out different factors associated with $\mathrm{CDH}$ recurrence including prenatal data as Lung to Head Ratio (LHR), postnatal clinical variables including gestational age (HBD), birth weight, Apgar score at birth, presence of pulmonary hypertension, use of Extracorporeal Membrane Oxygenation (ECMO), time of initial surgery, length of hospital stay or data connected directly with anatomy of the defect as side of hernia, presence of the hernia sac, size of the defect and need of a patch repair, liver herniation or type of the surgical approach (minimally invasive or classical). However, only small amount of $\mathrm{CDH}$ recurrences reports in literature describe large homogenous populations with a long follow-up, 1,3-6 most available data on $\mathrm{CDH}$ recurrence has been limited to small case series or are a multicenter study 7,8 including heterogenous cohorts. Our institutional approach to managing neonatal $\mathrm{CDH}$ has remained constant over the last 13 years. This has permitted us to query a large homogenous study population for the independent predictors of $\mathrm{CDH}$ recurrence.

\section{Materials and Methods}

The aim of this study is to asses the rate, risk-factors, morphology and treatment of $\mathrm{CDH}$ recurrence in homogenous series of $\mathrm{CDH}$ patients.

Medical records of 95 neonates with $\mathrm{CDH}$ who had undergone diaphragmatic hernia repair at tertiary pediatric teaching hospital, 
between 2007-2018 were retrospectively reviewed. All deaths $(\mathrm{N}=11)$ were excluded from the assessment. Data collected prior to the surgery included: demographics, antenatal or postnatal diagnosis, LHR, side of defect, gestational age, birth weight, Apgar scores at 1 st minute, presence of persistent pulmonary hypertension defined as High Frequency Oscillatory Ventilation (HFOV) and Inhaled Nitric Oxide (iNO) need of use, length of stabilization before the surgery described as the day of the surgery. All patients were stabilized before and after the surgical repair according to a standard protocol and practice guideline for $\mathrm{CDH}$ management at the hospital which is similar to Standard Postnatal Management of Infants with Congenital Diaphragmatic Hernia in Europe by The CDH EURO Consortium Consensus. All patients underwent laparotomy proceeded by the same $\mathrm{CDH}$ team. If the operator was a senior resident doctor, one of the $\mathrm{CDH}$ team surgeons was always assisting. The approach was via a subcostal incision on the ipsilateral side of hernia. After reduction of the abdominal viscera from the thorax, diaphragmatic defect was evaluated. In case of a small size of the defect (A or B by the CDH Study Group), the diaphragm was primarily closed with nonabsorbable suture. Large defects ( $\mathrm{C}$ or $\mathrm{D}$ by the CDH Study Group) were closed with a Gore-Tex patch tailored to restore a natural, tension-free diaphragm shape. In case of hernia sac presence, it was excised. If closure of abdominal wall was likely to increase intraabdominal pressure, SILO was used. Peri-operative data collected included: type of the diaphragm repair-primary suturing or prosthetic patch, presence of the hernia sac, hernia content (liver up), primary or delayed abdominal wall closure, postoperative length of ventilation (time of extubation), presence of persistent pulmonary hypertension defined as HFOV and iNO need of use. Recurrence data included: rate and age of the recurrence, its morphology (localization of re-herniation), content of hernia, symptoms and treatment (surgical or conservative). The follow-up was from 1 to 12 years after the initial repair. All of the patients underwent elective $\mathrm{x}$-ray in the first hour after the surgery, chest Computed Tomography (CT) scan before the discharge from the hospital, chest X-ray at the age of 6 months, chest CT scan at the age of 12 and 24 months of age, chest $\mathrm{x}$-ray at the age of 3 years. The patients were divided into the recurrence and non-recurrence groups. All the variables were compared between these two groups.

The statistical analyses were assessed by $x^{2}$ and MannWhitney -U tests using Statistica 10.0 Statsoft Poland. A p value of $£ 0.05$ was considered statistically significant.

\section{Results}

\section{Recurrence rate}

Recurrence occurred in 22/84 (26\%) patients. Additionally, in 5 of those patients we observed more than one recurrence, which gives the total number of 27 (32\%) re-herniations.

\section{Recurrence age}

Of all first recurrences 16/22 (72\%) occurred within first 12 months of the initial repair.

Other 9/27 recurrences (including multiple re-herniation) occurred by an age of 3 years, and only $2 / 27$ recurrences occurred after 3 year of age.

\section{Recurrence symptoms}

Seven out of all $27(26 \%)$ recurrences were symptomatic which among all 84 patients gives the symptomatic recurrence rate $8.3 \%$. Other $20 / 27(74 \%)$ recurrences were asymptomatic diagnosed during the planned CT scan control. Amongst patients with symptomatic recurrence in 3 cases symptoms were cough, dyspnea and respiratory failure, in 4 cases bowel obstruction. Six out of 7 symptomatic re-herniation occurred in the first year of life. In one patient who had two re-herniations, both were symptomatic, in one patient first recurrence was asymptomatic and the second one with symptoms of bowel obstruction. In all symptomatic cases content of re-herniation were intestines, defect was located postero-lateraly. All symptomatic patients were initially in a group with lower Apgar score and need of HFOV and iNO treatment.

\section{Demographics and prenatal-related factors comparison}

The majority of the neonates $68 / 82(83 \%)$ were diagnosed antenatally. Missing values about two patients with recurrence. Antenatal diagnosis was not a significant predictor of recurrence (Table 1).

Table 2 summarizes the comparison of perinatal factors between infants with and without recurrence. There were no significant differences between groups considering LHR, gestational age and birth weight. Significantly higher Apgar scores were in the group without recurrence.

Table 1. Prenatal diagnosis.

\begin{tabular}{|c|c|c|c|c|}
\hline Prenatal diagnosis & Recurrence & $\begin{array}{l}\text { YES } \\
\text { NO }\end{array}$ & $\begin{array}{c}17 \\
5\end{array}$ & $\mathrm{p}=0,62219$ \\
\hline & Non-recurrence & $\begin{array}{l}\text { YES } \\
\text { NO }\end{array}$ & $\begin{array}{c}51 \\
9\end{array}$ & \\
\hline
\end{tabular}

Table 2. Perinatal factors. LHR: lung to head ratio. HBD: gestational age.

\begin{tabular}{|c|c|c|c|c|c|c|c|}
\hline & & $\mathbf{N}$ & Mean value & Median value & Min & $\operatorname{Max}$ & p-value \\
\hline \multirow[t]{2}{*}{ LHR } & Recurrence & 13 & 1,47 & 1,49 & 0,9 & 2,2 & \multirow[t]{2}{*}{0,340338} \\
\hline & Non-recurence & 37 & 1,57 & 1,6 & 0,4 & 2,6 & \\
\hline \multirow[t]{2}{*}{ HBD } & Recurrence & 21 & 37,8 & 38 & 33 & 41 & \multirow[t]{2}{*}{0,535626} \\
\hline & Non-recurrence & 61 & 37,5 & 38 & 31 & 41 & \\
\hline \multirow[t]{2}{*}{ Birth weight (g) } & Recurrence & 20 & 3159 & 3170 & 1820 & 4020 & \multirow[t]{2}{*}{0,607928} \\
\hline & Non-recurrence & 62 & 3089 & 3125 & 1300 & 4220 & \\
\hline Apgar score & $\begin{array}{l}\text { Recurrence } \\
\text { Non-recurrence }\end{array}$ & $\begin{array}{l}22 \\
61\end{array}$ & $\begin{array}{l}5,9 \\
7,1\end{array}$ & $\begin{array}{l}6 \\
7\end{array}$ & $\begin{array}{l}1 \\
2\end{array}$ & $\begin{array}{l}10 \\
10\end{array}$ & 0,030209 \\
\hline
\end{tabular}




\section{Prior to surgery related factors comparison}

Table 3 and 4 show the comparison between children who developed recurrence and children who did not in terms of the length of pre-surgery cardiopulmonary stabilization described as a day of the initial surgery and presence of persistent pulmonary hypertension defined as HFOV and iNO need of use during that period of time.

All the factors connected with severity of pre-surgery stabilization period turned out not to be considered as significantly important factors of re-herniation in $\mathrm{CDH}$ patients.

\section{CDH anatomy connected factors comparison}

Among subjects with recurrent $\mathrm{CDH}$ majority had a left-sided defect, hernia sac was not present, liver was not herniated into the thorax, diaphragm was primarily sutured and abdominal wall primarily closed. Even though none of those factors was significantly related with the $\mathrm{CDH}$ recurrence (Table 5).

\section{Post-surgery factors comparison}

Table 6 and 7 show the comparison between children who developed recurrence and children who did not in terms of the length of post-surgery cardiopulmonary stabilization described as a day of extubation and presence of persistent pulmonary hyper- tension defined as HFOV and iNO need of use during that period of time.

All the factors connected with severity of post-operative stabilization period turned out not to be considered as significantly important factors of re-herniation in $\mathrm{CDH}$ patients. However, the need of HFOV after the surgery approached significance $(p=0.06)$.

\section{Diagnosis, morphology and treatment of recurrence}

The diagnosis of symptomatic recurrences was confirmed with an x-ray (Figure 1). All diagnosis of asymptomatic re-herniations were based on elective CT (Figure 2). In 25/27 (92.5\%) cases of $\mathrm{CDH}$ recurrence the defect of the diaphragm was located in the postero-lateral part of the repaired diaphragm (Figure 3), in one case (3\%) medially (Figure 4), in one case (3\%) we observed diaphragm relaxation after primarily repaired defect without hernia sac. In $23 / 27(85 \%)$ recurrences the content of hernia were intestines (Figure 1 and 3), in 3/27 (11\%) kidney (Figure 5), in one (3\%) part of the stomach (Figure 4). Among symptomatic re-herniations all the patients were operated as an urgent procedure. Patients with asymptomatic recurrence and bowel herniated into the chest were qualified for elective surgery. In one case parents declining procedure for asymptomatic re-herniation, the child stays asymptomatic carefully monitored. In three cases of asymptomatic kidney re-herniation, all

Table 3. Time of the initial surgical repair-length of initial cardiopulmonary stabilization.

\begin{tabular}{|c|c|c|c|c|c|c|c|}
\hline & & $\mathbf{N}$ & $\begin{array}{l}\text { Mean } \\
\text { value }\end{array}$ & $\begin{array}{l}\text { Median } \\
\text { value }\end{array}$ & Min & Max & p-value \\
\hline $\begin{array}{l}\text { Age at the repair } \\
\text { (days of life) }\end{array}$ & $\begin{array}{l}\text { Recurrence } \\
\text { Non-recurrence }\end{array}$ & $\begin{array}{l}21 \\
62\end{array}$ & $\begin{array}{l}5,3 \\
4,2\end{array}$ & $\begin{array}{l}3 \\
3\end{array}$ & $\begin{array}{l}1 \\
1\end{array}$ & $\begin{array}{l}15 \\
28\end{array}$ & 0,416480 \\
\hline
\end{tabular}

Table 4. High Frequency Oscillatory Ventilation (HFOV) and Inhaled Nitric Oxide (iNO) need of use in initial cardiopulmonary stabilization.

\begin{tabular}{llll} 
& & $\mathbf{N}(\%)$ & P-value \\
HFOV & Recurrence & $20(90,91 \%)$ & 0,14189 \\
prior-surgery & Non-recurrence & $45(72,58 \%)$ & \\
iNO & Recurrence & $15(68,18 \%)$ & 0,22328 \\
prior-surgery & Non-recurrence & $33(53,23 \%)$ & \\
\hline
\end{tabular}

Table 5. Anatomy of the defect.

\begin{tabular}{|c|c|c|c|c|}
\hline Defect side & $\begin{array}{l}\text { Recurrence } \\
\text { Non-recurrence }\end{array}$ & $\begin{array}{l}\text { Left } \\
\text { Right } \\
\text { Left } \\
\text { Right }\end{array}$ & $\begin{array}{c}20 \\
2 \\
54 \\
8\end{array}$ & $\mathrm{p}=0,92731$ \\
\hline Hernia sac presence & $\begin{array}{l}\text { Recurrence } \\
\text { Non-recurrence }\end{array}$ & $\begin{array}{l}\text { YES } \\
\text { NO } \\
\text { YES } \\
\text { NO }\end{array}$ & $\begin{array}{c}4 \\
18 \\
19 \\
43\end{array}$ & $\mathrm{p}=0,26004$ \\
\hline Liver-up & $\begin{array}{l}\text { Recurrence } \\
\text { Non-recurrence }\end{array}$ & $\begin{array}{l}\text { YES } \\
\text { NO } \\
\text { YES } \\
\text { NO }\end{array}$ & $\begin{array}{l}7 \\
15 \\
22 \\
40\end{array}$ & $\mathrm{p}=0,75604$ \\
\hline Patch repair & $\begin{array}{l}\text { Recurrence } \\
\text { Non-recurrence }\end{array}$ & $\begin{array}{l}\text { Primary } \\
\text { GoreTex } \\
\text { Primary } \\
\text { GoreTex }\end{array}$ & $\begin{array}{c}15 \\
7 \\
50 \\
12\end{array}$ & $\mathrm{p}=0,36606$ \\
\hline Abdominal wall closure & $\begin{array}{l}\text { Recurrence } \\
\text { Non-recurrence }\end{array}$ & $\begin{array}{l}\text { Primary } \\
\text { SILO } \\
\text { Primary } \\
\text { SILO }\end{array}$ & $\begin{array}{c}20 \\
2 \\
55 \\
7\end{array}$ & $\mathrm{p}=0,90875$ \\
\hline
\end{tabular}


of the patients were treated expectantly while carefully monitored for kidneys function. An open procedure was performed in all recurrences. In 4 cases, where initially the diaphragm defect was primarily sutured, recurrences were treated with a patch. The children who had multiple recurrences, had recurrences repaired with a patch each time. In case of relaxation of primarily sutured diaphragm, plication during the repair of the recurrence was proceeded.

\section{Characteristic of the multiple recurrence group}

In 5 of 22 patients we observed more than one recurrence. In three of those patients first recurrence, in two cases also second reherniation was symptomatic. In 4 of those 5 patients during the first surgery, because of a large defect Gore-Tex patch was used, in one case diaphragm was sutured primarily. All the re-herniations in this group were closed with a patch. In all cases, during all recurrences the defect was located postero-lateral and contents were intestines. All those patients were diagnosed prenatally, needed HFOV and iNO before and after the first surgery, none of them needed it before or after the recurrence.

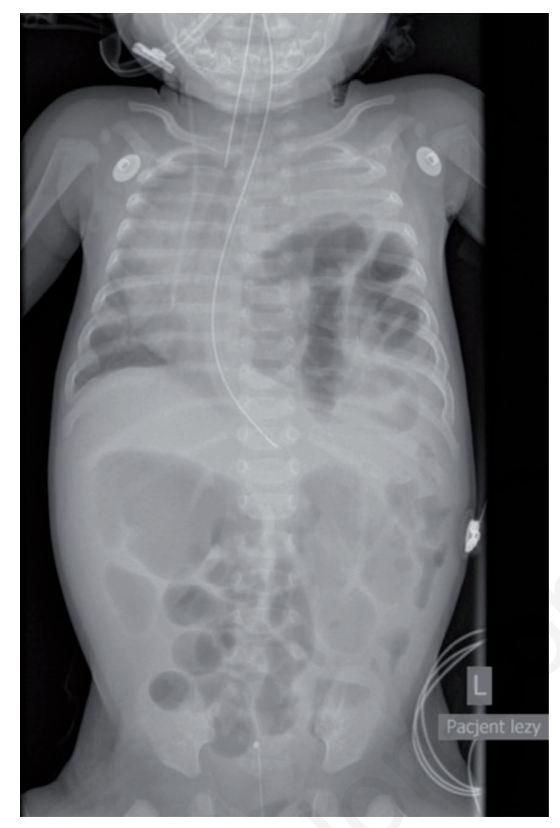

Figure 1. Symptomatic recurrences confirmed with an $x$-ray. Intestines as a content of hernia.

\section{Discussion}

Recurrence of a diaphragmatic hernia is among pulmonary hypoplasia, pulmonary hypertension, gastroesphageal reflux, failure to thrive, bowel obstruction, chest deformities one of the known and accepted complications following successful initial repair. The reported incidence of re-herniation after a $\mathrm{CDH}$ repair has varied from $2-50 \%, 1,3-6,8-13$ The reason for such a wide range is unclear, in our opinion, it is almost certainly caused by the lack of structured follow-up in different institutions as well as ununiform $\mathrm{CDH}$ re-herniations reporting in the literature. Some of the studies draw conclusions only from symptomatic recurrences ${ }^{1}$ or report only early re-herniations, ${ }^{7}$ some studies forewarn that in their material routine $\mathrm{x}$-ray screening is not standard during the follow-up $1,3,8$ which in a significant way underestimates the actual $\mathrm{CDH}$ recurrences rate. In this study we report one of the largest cohorts of a single institution with the long, homogenous, precise and intensified follow-up protocol which gave us opportunity to detect all symptomatic and asymptomatic, early and late recurrences.

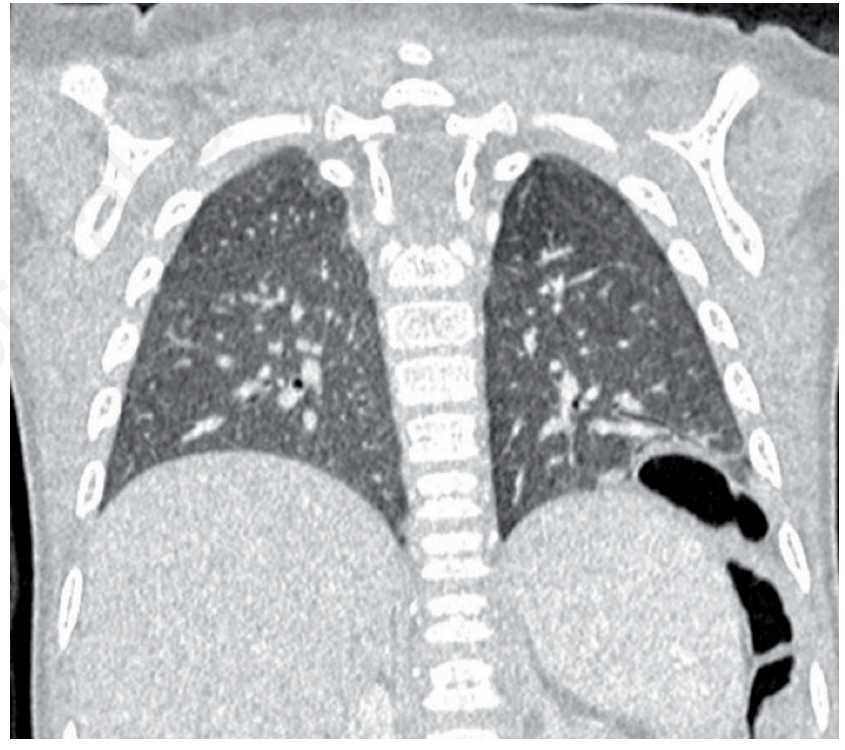

Figure 2. Asymptomatic re-herniations in elective CT.

Table 6. Extubation time.

\begin{tabular}{llcccccc} 
& & N & Mean & Median & Min & Max & P-value \\
value & value & & & & \\
Extubation time & Recurrence & 20 & 9 & 7,5 & 1 & 25 & 0,313507 \\
& Non-recurrence & 59 & 7 & 6 & 1 & 28 & \\
\hline
\end{tabular}

Table 7. High Frequency Oscillatory Ventilation (HFOV) and Inhaled Nitric Oxide (iNO) need of use in post-operative cardiopulmonary stabilization.

\begin{tabular}{llll} 
& & $\mathbf{N}(\%)$ & p \\
HFOV & Recurrence & $10(45,45)$ & $\mathrm{p}=0,49045$ \\
post-surgery & Non-recurrence & $37(59,68)$ & \\
iNO & Recurrence & $10(45,45)$ & $\mathrm{p}=0,49045$ \\
post-suregry & Non-recurrence & $23(37,10)$ & \\
\hline
\end{tabular}



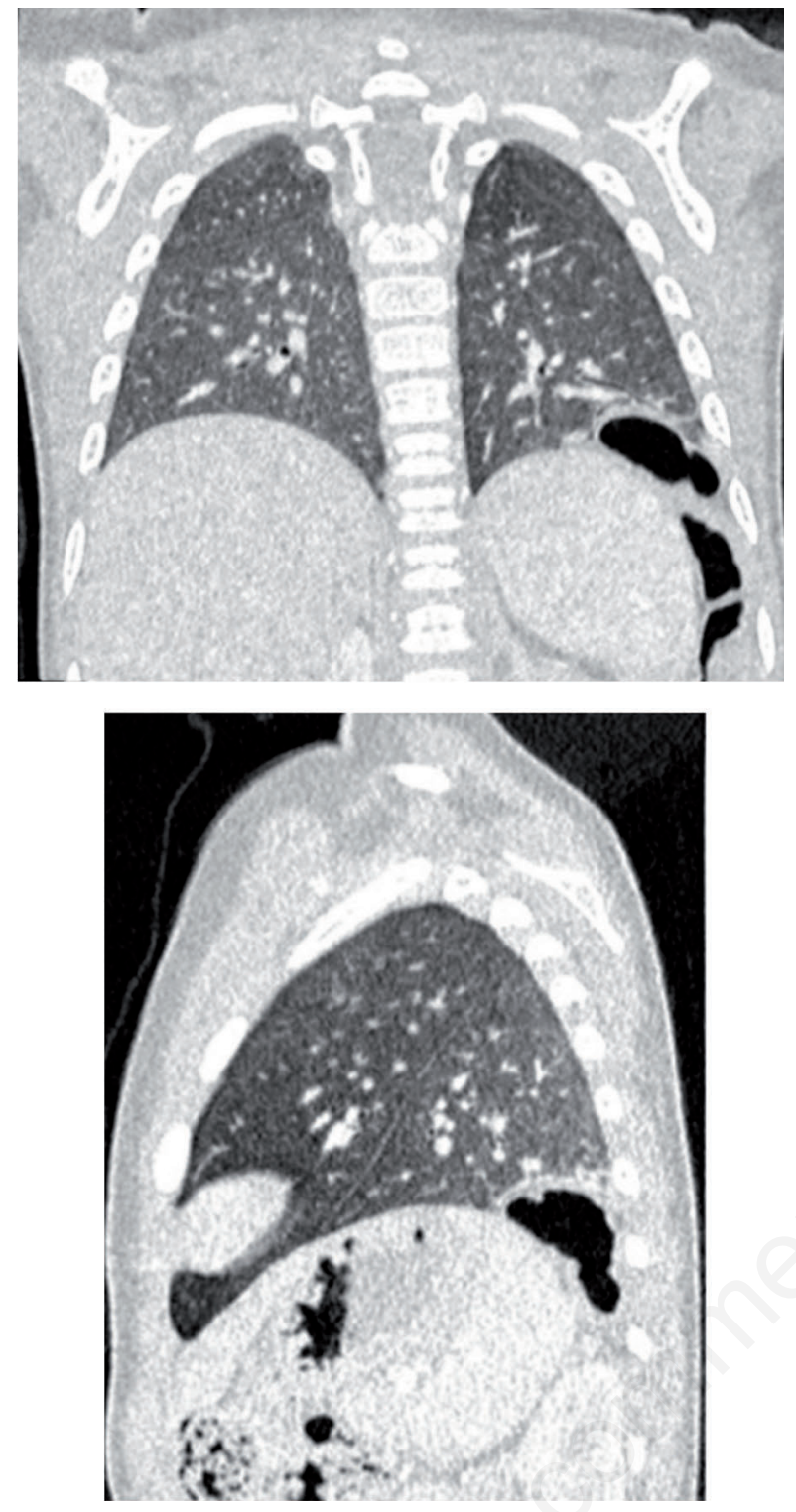

Figure 3. $\mathrm{CDH}$ recurrence located in the postero-lateral part of the repaired diaphragm. Intestines as a content of hernia.

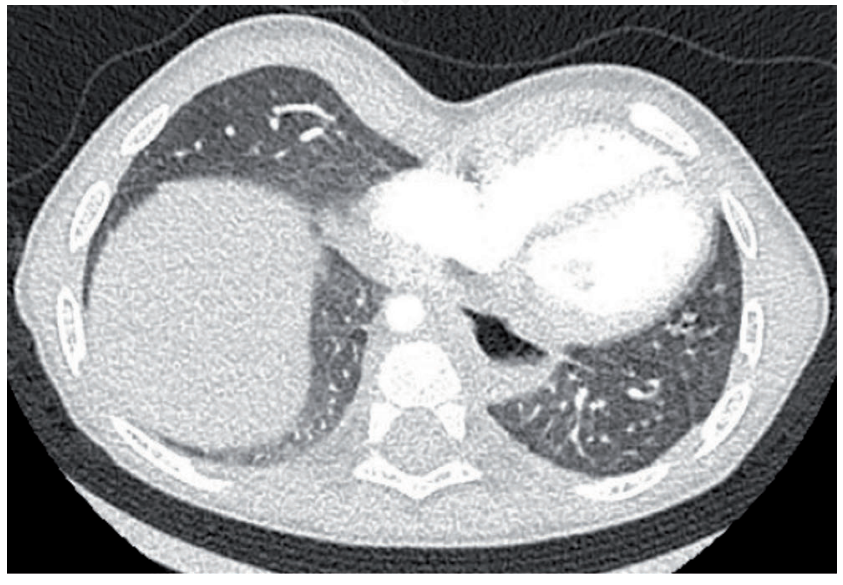

Figure 4. $\mathrm{CDH}$ recurrence located in the medial part of the repaired diaphragm. Stomach as a content of hernia.
A diaphragmatic hernia can reoccur at any time, but the majority present within the first 12 or 24 months of life.1,3,5-8,11 We too have noted that most reherniations occur prior to 12 months of age, and have tailored our screening program accordingly.

Patients with re-herniation can be divided into two groups: symptomatic and asymptomatic. The second group is possible to detect only with intensified follow-up protocol. Otherwise, asymptomatic recurrences remain unknown blurring the re-herniation rate. In our material symptomatic recurrences constitute only $26 \%$ of all re-herniations which gives $8.3 \%$ among all 84 patients. Patients who present with symptoms related to a re-herniation generally fall into two categories: those with pulmonary and those with gastrointestinal symptoms, ${ }^{9}$ which we observed also in our patients.

We have investigated whether factors widely known as associated with the risk of poorer survival rate and higher morbidity would play a role in predicting $\mathrm{CDH}$ recurrence. Among most important prenatal-patient related factors (Table 1 and 2) only Apgar score was independent predictor of re-herniation being significantly lower in recurrence group which is in line with previous series of Al-lede et al. ${ }^{1}$ and Putman et al. ${ }^{7}$ However, in many stud-
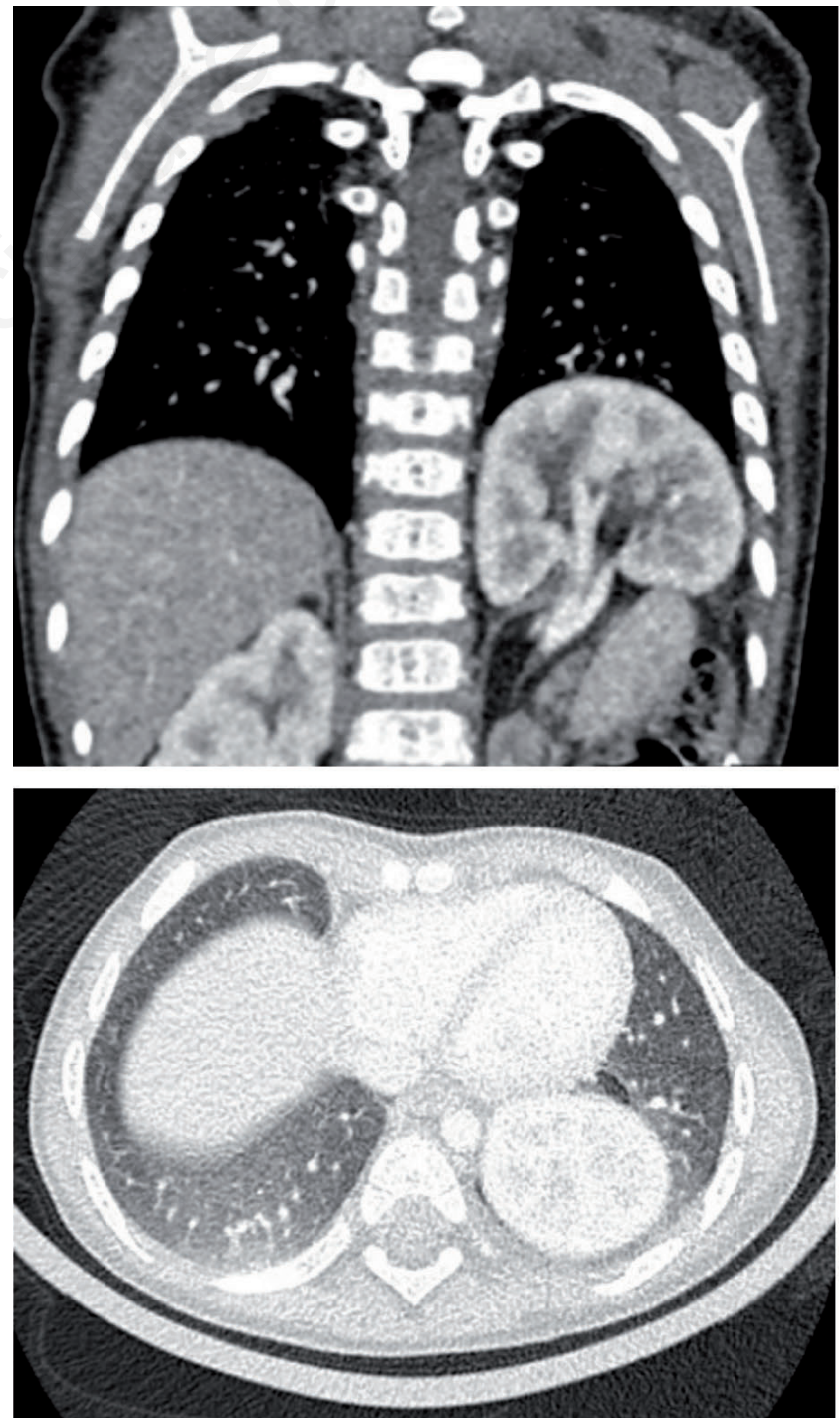

Figure 5. Kidney as a content of hernia. 
ies including two large-volume studies by Nagata et al. and Fisher et al. we find suggestion that prior to birth, all surviving neonates may carry a similar risk of recurrence. 5,8 Indeed, we did not detect significant differences between neonates with and without $\mathrm{CDH}$ recurrence in any of other perinatal factors.

While many large-volume studies have emphasized the role of patch repair in recurrence risk stratification this finding was not confirmed in our series. What is more, in contrast to previous studies, any other factors connected with the anatomy of $\mathrm{CDH}$ (side, hernia sac presence, liver up, type of abdominal wall closure) were not detected as independent re-herniation risk factors. ${ }^{1-1}$ There is no clear explanation for this fact found in our material.

The comparison between children who developed recurrence and children who did not in terms of the length of pre-and postsurgery cardiopulmonary stabilization described as a day of the initial surgery, day of extubation and presence of persistent pulmonary hypertension defined as HFOV and iNO need of use during that period of time turned out not to be considered as significantly important factors of re-herniation in $\mathrm{CDH}$ patients in our series. However, the need of HFOV after the surgery approached significance $(p=0.06)$. Our analysis stays in contrast to series described by Al-lade et al. showing that children developing recurrence were more likely to require HFOV and iNO prior to surgery, post-operatively required sildenafil, longer duration of mechanical ventilation and oxygen supplementation.

Taken together, these data suggest that neonates in this study at risk for $\mathrm{CDH}$ recurrence were best identified by severe clinical manifestation, i.e. lower Apgar score at birth and the need of HFOV after the surgery. This recurrence risk appears independent of other previously reported factors.

The diagnosis of symptomatic recurrences was confirmed with only an X-ray (AP and lateral view). All diagnosis of asymptomatic re-herniations were based on elective CT which helped to better understand the anatomy as regards the location of re-herniation and the amount of abdominal contents involved. Once it has established that the re-herniation has occurred, symptomatic patients should be urgently repaired. However, in asymptomatic recurrence with a small defect, patients can be treated expectantly for several months being carefully monitored which allows child to grow for a more secure repair later and fewer repair in total.

What seem to be worth mentioning are three recurrences with the kidney as a content of the hernia. In one case in preoperative ultrasound examination there was an intrathoracic kidney detected. In other two cases kidneys were observed perioperatively above its normal location before closure of the diaphragm. Urinary system anomalies affect approximately $10 \%$ of population. The thorax is an extremely rare state of aberrant kidney. Nearly $5 \%$ of all ectopic kidneys are intrathoracic. The majority of patients with an intrathoracic kidney are asymptomatic and their kidney functions are almost always normal therefore does not require any specific treatment. 13 Intrathoracic kidneys are divided into four distinct groups based on the status of the diaphragm: i) Group 1: Intrathoracic ectopic kidney associated with a closed diaphragm; ii) Group 2: Intrathoracic ectopic kidney associated with diaphragmatic eventration; iii) Group 3: Intrathoracic ectopic kidney associated with traumatic diaphragmatic rupture; and iv) Group 4: Intrathoracic ectopic kidney associated with a congenital diaphragmatic hernia. An intrathoracic ectopic kidney associated with a Bochdalek hernia is a very rare condition with a reported incidence of $0.25 \% .14$ That is why in those cases recurrence may be result of failure of the initial repair and as long as the hernia content is only an ectopic normally functioning kidney, the patients can be treated expectantly.

\section{Conclusions}

In conclusion, recurrence in $\mathrm{CDH}$ remains a significant issue for long-term surgical morbidity, especially more apparent in the first year of life. As long as the risk factors of re-herniation remains unclear and the majority of re-herniations are asymptomatic, crucial seems to be routine follow-up protocol allowing for $\mathrm{CDH}$ recurrence detection. Important is to recognize respiratory and digestive symptoms as manifestation of a possible $\mathrm{CDH}$ recurrence requiring urgent surgery. In case of asymptomatic re-herniation of intestines or stomach, elective surgery seems to be the treatment of choice. In case of re-herniated kidney expectant approach with careful monitoring for kidneys function may be reasonable.

\section{References}

1. Al-lede MM, Karpelowsky J, Fitzgerald DA. Recurrent Diaphragmatic Hernia: Modifiable and Non-Modifable Risk Factors. Ped Pulmonology 2016;51:394-401.

2. Rowe DH, Stolar CJ. Recurrent diaphragmatic hernia. Semin Ped Surg 2003;12:107-9.

3. Janssen S, Hewegen K, van Rooij IA, et al. Factors related to long-term surgical morbidity in congenital diaphragmatic hernia survivors. J Ped Surg 2018;53:508-12.

4. Jancelewicz T, Chiang M, Oliveira C, Chiu PP. Late surgical outcomes among congenital diaphragmatic hernia $(\mathrm{CDH})$ patients: why long-term follow-up with surgeons is recommended. J Ped Surg 2013;48:935-41.

5. Fisher JC, Haley MJ, Ruiz-Elizalde A, et al. A multivariate model for predicting recurrence in congenital diaphragmatic hernia. J Ped Surg 2009;44:1173-80.

6. Moss RL, Chen CM, Harrison MR. Prosthetic patch durability in congenital diaphragmatic hernia: a long-term follow-up study. J Ped Surg 2001;36:152-4.

7. Putman LR, Gupta V, Tsao K, et al. Factors associated with early recurrence after congenital diaphragmatic hernia repair. J Ped Surg 2017;52:928-32.

8. Nagata K, Usui N, Terui K, et al. Risk Factors for the Recurrence of the Congenital Diaphragmatic Hernia-Report from Long-Term Follow-Up Study of Japanese CDH Study Group. Eur J Ped Surg 2015;25:9-14.

9. Hayward ML, Fauza DO, Wilson JM. Complications of CDH Repair and Recurrent CDH. In: Teich S, Caniano DA (eds). Reoperative Pediatric Surgery. Humana Press; 2008.

10. Short HL, Clifton MS, Arps K, et al. The "Flat Diaphragm":Does the Degree of Curvature of the Diaphragm on Postoperative X-Ray Predict Congenital Diaphragmatic Hernia Recurrence? J Laparoendoscopic Advanced Surg Tech 2017;28:476-80.

11. Hajer GF, vd Staak FH, de Haan AF, Festen C. Reccurent congenital diaphragmatic hernia: which factors are involved? Eur J Ped Surg 1998;8:329-33.

12. Brindle ME, Brar M, Skarsgard ED. Patch repair is an independent predictor of morbidity and mortality in congenital diaphragmatic hernia. Ped Surg Int 2011;27:969-74.

13. Khoshchehreh M, Paknejad O, Bakhshayesh-Karam M, Pazoki M. Thoracic Kidney: Extremely Rare State of Aberrant Kidney. Case Rep Urol 2015:672628.

14. Sarac M, Bakal U, Tartar T, et al. Bochdalek hernia and intrathoracic ectopic kidney: Presentation of two case reports and review of the literature. Niger J Clin Pract 2018;21:681-6. 